\title{
Recent Clinical, Biochemical and Radiological Factors Affecting Outcomes of Diabetic Foot Ulcer
}

\author{
Arijit Singha Mahapatra ${ }^{1}$, Pritam Mitra $^{2 *}$
}

\author{
${ }^{1}$ Consultant, Department of Surgery, M. R Bangur Dist Hospital, 241, Deshpran Sasmal Rd, Netajinagar, Rajendra Prasad Colony, \\ Tollygunge, Kolkata, West Bengal 700033, India \\ ${ }^{2}$ Medical Officer, Department of Surgery, M. R Bangur District Hospital, 241, Deshpran Sasmal Rd, Netajinagar, Rajendra Prasad \\ Colony, Tollygunge, Kolkata, West Bengal 700033, India
}

DOI: $\underline{10.36347 / \text { sasjs.2021.v07i03.004 }}$

| Received: 23.02.2021 | Accepted: 04.03.2021 | Published: 08.03.2021

*Corresponding author: Pritam Mitra

\section{Abstract}

Original Research Article

Introduction: Diabetic foot ulcer (DFU) is the most costly and devastating complication of diabetes mellitus, which affect $15 \%$ of diabetic patients during their lifetime. Early and effective management of DFU can reduce the severity of complications such as preventable amputations and possible mortality, and also can improve overall quality of life. Aim of study: To study the clinical, biochemical and radiological factors affecting outcomes of diabetic foot ulcer. Methods: A prospective observational study was conducted between august, 2019 and July, 2020. All diagnosed cases of diabetic foot ulcer attending OPD or admitted in the Department of General Surgery were included in this study. These cases were followed through the entire course from diagnosis to outcomes. All the clinical, biochemical and radiological findings were recorded and evaluated statistically. Results: A total of 60 patients were admitted with diagnosis of diabetic foot ulcer during this duration. Thirty two percent of patients admitted were between $61-70$ years old. Male to female ratio was 10:3. Toe gangrene (29\%) followed by infected ulcer $(25 \%)$ was the most common clinical presentation. History of trauma was present in $42 \%$ of patients. Twenty eight percent of patients had diabetes for 6-10 years. Most common etiological factor was neuro-ischemic i.e 18/60 patients. Smoking (46\%) followed by trauma $(40 \%)$ were the commonest risk factors. Staph aureus was the infecting organism in 16 cases. Peripheral vascular disease was present in $51 \%$ of the cases. Out of which 28 cases were male and 2 were female. Conclusion: Incidence of diabetic foot ulcer increases with age. Ulceration, infection and gangrene are the commonest presentation. Prevention of smoking and foot care program with multidisciplinary team can prevent DFU and its complications.

Keywords: Diabetic footulcer, trauma, foot infection.

Copyright $(\mathcal{C} 2021$ The Author(s): This is an open-access article distributed under the terms of the Creative Commons Attribution 4.0 International License (CC BY-NC 4.0) which permits unrestricted use, distribution, and reproduction in any medium for non-commercial use provided the original author and source are credited.

\section{INTRODUCTION}

Diabetic ulcer disease, a major complication of DM, has gained importance because of the socioeconomic burden it imposes on the patient, the patient's family and on the health care system of the country. India is considered as the Diabetic capital of the world because of its high prevalence [1]. Diabetic foot syndrome (DFS) is one of the chronic complications of diabetes. The early detection of peripheral vascular disease in asymptomatic and early cases is useful in improving the blood flow and hence healing and reduction of risk of major limb amputations. It is estimated that approximately 50-70\% of all lower limb amputations are due to diabetic foot ulcer (DFU) and in addition it is also reported that in every $30 \mathrm{sec}$ one leg is amputated due to DFU in worldwide [2, 3]. There is a need for proper systemic evaluation of peripheral vascular disease in all diabetic patients especially patients presenting with diabetic foot infections. Aim of this study is to find out etiology, risk factors and prevalence of peripheral vascular disease in DFU and with the help of above mentioned parameters, to find out factors that are affecting the outcome of DFUs significantly.

\section{METHOD}

This prospective observational study was conducted in a tertiary care center august, 2019 and July, 2020. All diagnosed cases of diabetic foot ulcer attending OPD or admitted in the Department of General Surgery were included in this study. Diagnosed cases of DFU admitted in the department of general surgery were included in the study. Patients above 80 Years of age with cardiac, renal diseases \& other 
comorbid conditions and juvenile diabetes mellitus were excluded. Parameters studied were etiology i.e neuropathic, ischemic and neuro-ischemic, risk factors - mechanical stress, trauma, smoking, nutritional status, CRF, location of ulcers, contaminating organisms, presence of peripheral vascular disease.

Total of 60 cases were enrolled in this study during the time period. These cases were then followed up for 6 months. Cases were further defined as healed and non-healed ulcer depending on whether the diabetic foot ulcer was healed in this time period. These 2 groups were further analysed for association with different factors. Statistical analysis was conducted with the statistical package for the social science system (SPSS) version 17.0.

\section{RESULTS}

Analysis of 60 cases of diabetic foot was done. Most of the patients were between age group 61- 70 years $(30 \%)$ followed by 71-80 (24\%). Male: female ratio was $3: 1$. Twenty four patients had infected ulcer, 20 patients had abscess and 16 patients had gangrene.

In this study, most common etiology found out to be neuroischaemic $(41.6 \%) .33 .3 \%$ patients were presented with neuropathic DFU. Exclusively, ischaemia was cause in $25 \%$ of cases. Most common site of ulcer was foot margin $(26.67 \%)$, followed by plantar surface of metatarsal head and toes tip. Among the 39 cases having a positive growth in culture, the most common contaminating organism was staph aureus $(26.7 \%)$, followed by pseudomonas aeruginosa $(10 \%)$. In $20.2 \%$ cases contaminating organisms were polymicrobial. $35 \%$ cases showed no growth in culture. It was observed that history of smoking is associated in $46.67 \%, \mathrm{H} / \mathrm{O}$ hypertension in $48.3 \%$, raised HBA1C in $23.3 \%$, altered lipid profile in $30 \%$, H/O poor foot wear in $20 \%$, limb deformity in $10 \%$, and restricted joint mobility in $8.33 \%$.

Out of 60 patients in this study, 50\% were found to have some form of reduced arterial flow of the lower limb vessels. Among them33.3\% were found to be smoker. Distal calf vessels were involved in $90 \%$ of patients, with both vessels in $50 \%$ and distal vessels in $40 \%$ of patients found to have peripheral arterial compromise. Six (10\%) of patients had retinopathy, $11.6 \%$ and $6.66 \%$ had history of CAD and CVA respectively. Nine $(15 \%)$ cases had nephropathy and $5 \%$ had history of dialysis. At 6 months of follow up, 46 cases $(76.7 \%)$ were healed, and $14(23.3 \%)$ were not healed.

The cases were further divided in two groups, group I consisting of non-healing ulcer (14 cases) and group II consisting of healed ulcer (46 cases). There was significant relation between age and healing of ulcer $(\mathrm{p}-0.003)$. There is no significant association between gender and ulcer healing $(p-0.72)$. No significant association was found between site of ulcer and healing $(\mathrm{p}-0.82)$. Presence of hypertension affected ulcer healing $(\mathrm{p}-0.3)$. There was no association found between presences of co-morbidities like hyperlipidemia, CKD. Smoking was a significant factor for healing. Healing of ulcer was not significantly associated with etiology of the ulcers $(p-0.7)$. Diabetic status and serum albumin level directly affected healing $(\mathrm{p}-0.012)$.

Table-1: Showing demography of the study population

\begin{tabular}{|l|l|}
\hline Variables & $\mathbf{n}(\mathbf{\%})$ \\
\hline Age (years) & \\
\hline $31-40$ & $7(11 \%)$ \\
\hline $41-50$ & $8(13.3 \%)$ \\
\hline $51-60$ & $12(20 \%)$ \\
\hline $61-70$ & $20(33.3 \%)$ \\
\hline $71-80$ & $13(21.6 \%)$ \\
\hline $31-40$ & $7(11 \%)$ \\
\hline Sex & \\
\hline Male & $46(78 \%)$ \\
\hline Female & $14(22 \%)$ \\
\hline
\end{tabular}

Table-2: Distribution of cases according to presentation, etiology and site of ulcer

\begin{tabular}{|l|l|}
\hline Variables & $\mathbf{n}(\boldsymbol{\%})$ \\
\hline Clinical Presentations & \\
\hline Cellulitis & 26 \\
\hline Abscess & 20 \\
\hline Infected ulcer & 24 \\
\hline Gangrene & 16 \\
\hline Etiology & \\
\hline Neuropathy & $20(33.3 \%)$ \\
\hline Ischemia & $15(25 \%)$ \\
\hline Neuro-ischemic & $25(41.6 \%)$ \\
\hline Site of ulcer & \\
\hline Foot margin & $16(26.67 \%)$ \\
\hline Toes tip & $10(16.6 \%)$ \\
\hline Heel & $7(11.66 \%)$ \\
\hline Plantar surface of toes & $8(13.3 \%)$ \\
\hline $\begin{array}{l}\text { Plantar surface of metatarsals } \\
\text { head }\end{array}$ & $11(18.33 \%)$ \\
\hline Others & $8(13.3 \%)$ \\
\hline
\end{tabular}

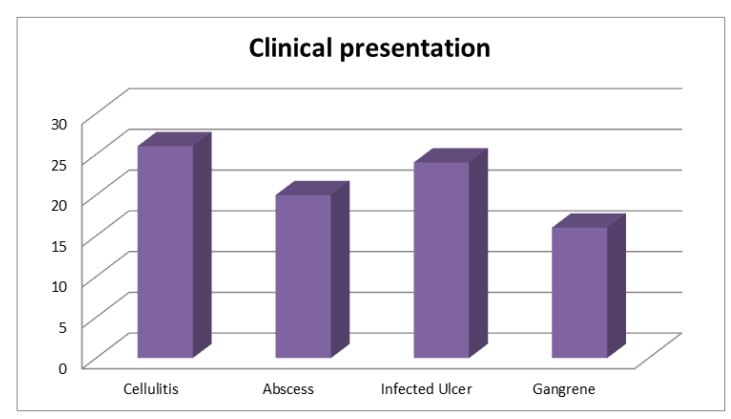

Bar graph showing distribution of clinical presentations 


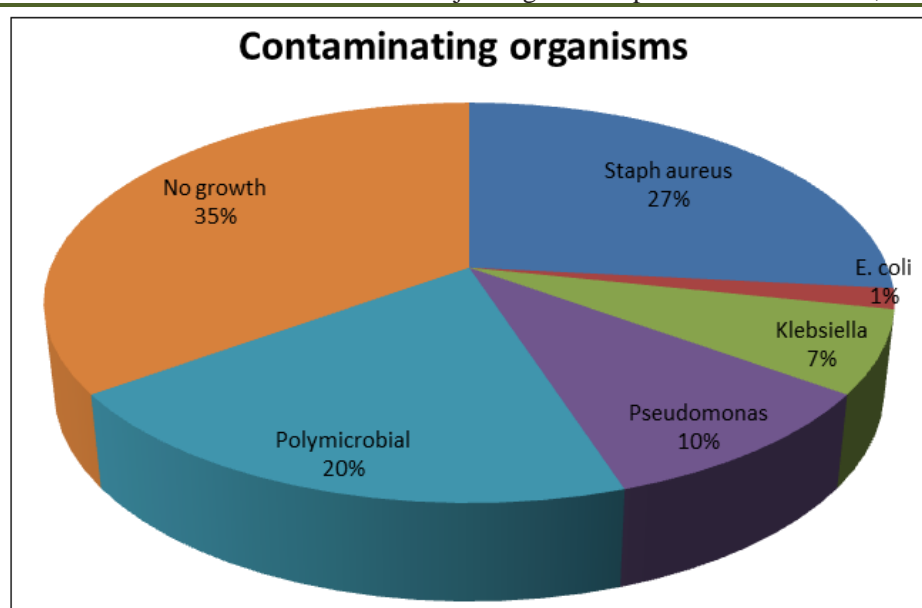

Fig-1: Pie chart showing prevalence of contaminating organisms

Table-3: Distribution of cases according to associations, PVD and complications

\begin{tabular}{|l|l|}
\hline Hypertensive & $\mathbf{2 9}(\mathbf{4 8 . 3} \%)$ \\
\hline HbA1c (mmol/mol) & 7 \\
$6-7$ & 39 \\
$7-9$ & $14(23.34 \%)$ \\
$>9$ & $18(30 \%)$ \\
\hline Altered lipid profile & 20 \\
\hline Poor foot wear & 6 \\
\hline Limb deformity & 5 \\
\hline Restricted joint mobility & $28(46.67 \%)$ \\
\hline Smoking & $30(50 \%)$ \\
\hline Smokers with PVD & $20(33.3 \%)$ \\
\hline Present & $10(16 \%)$ \\
\hline Absent & $6(10 \%)$ \\
\hline Complication Retinopathy & $7(11.6 \%)$ \\
\hline CAD & $4(6.66 \%)$ \\
\hline CVA & $9(15 \%)$ \\
\hline Nephrotoxicity & $3(5 \%)$ \\
\hline H/O dialysis & $6(10 \%)$ \\
\hline Retinopathy & $7(11.6 \%)$ \\
\hline CAD & \\
\hline
\end{tabular}

Table-4: Distribution of cases according to healing of ulcer

\begin{tabular}{|l|l|}
\hline Healed ulcer & $46(76.7 \%)$ \\
\hline Non healed ulcer & $14(23.3 \%)$ \\
\hline
\end{tabular}

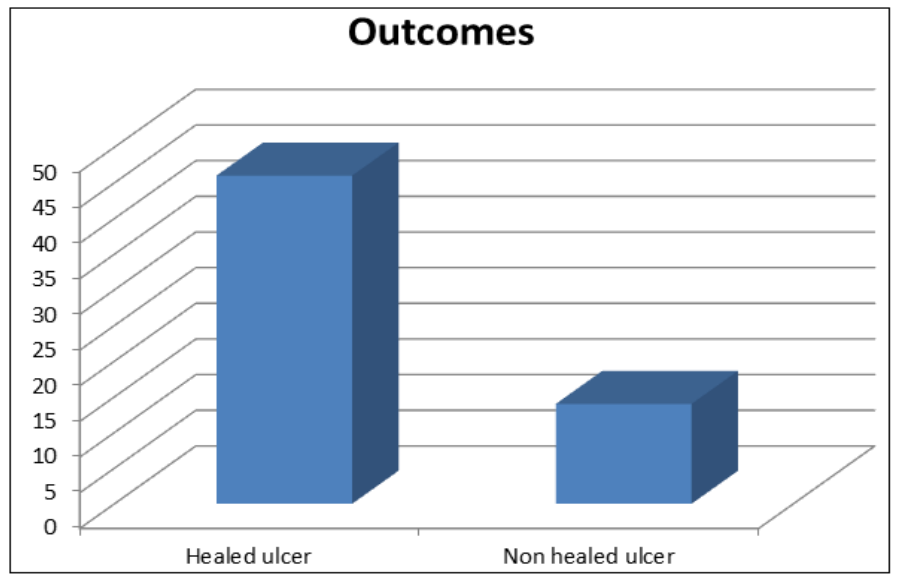

Fig-2 
Arijit Singha Mahapatra \& Pritam Mitra., SAS J Surg, Mar, 2021; 7(3): 124-129

Table-5: Distribution of cases in both the groups according to age, sex, presence of gangrene, site of ulcer

\begin{tabular}{|l|l|l|l|}
\hline Variables & $\begin{array}{l}\text { Group I (Non } \\
\text { healed ulcer n= 14) }\end{array}$ & $\begin{array}{l}\text { Group II (Healed } \\
\text { ulcer n = 46) }\end{array}$ & P value \\
\hline Age & & & \\
\hline $40-60$ & 1 & 11 & 0.003 \\
\hline $61-70$ & 5 & 15 & \\
\hline $71-80$ & 8 & 5 & \\
\hline $40-60$ & 1 & 11 & \\
\hline $61-70$ & 5 & 15 & \\
\hline Sex & & & 0.72 \\
\hline Male & 10 & 36 & \\
\hline Female & 4 & 10 & 0.0001 \\
\hline Gangrene & & & \\
\hline Present & 10 & 6 & 0.82 \\
\hline Absent & 4 & 40 & \\
\hline Site of ulcer & & & \\
\hline Toes tip & 4 & 6 & \\
\hline Foot margin & 4 & 12 & \\
\hline Forefoot & 2 & 0 & \\
\hline Plantar surface of metatarsal head & 1 & 10 & \\
\hline Dorsum of foot & 3 & 3 & \\
\hline Toes tip & 4 & 6 & 12 \\
\hline Foot margin & 4 & & \\
\hline
\end{tabular}

Table-6: Distribution of cases in 2 groups according to presence of co-morbidities, aetiology and bone changes

\begin{tabular}{|l|l|l|l|}
\hline Variables & $\begin{array}{l}\text { Group I ( Non healed } \\
\text { ulcer n= 14) }\end{array}$ & $\begin{array}{l}\text { Group II (Healed } \\
\text { ulcer n = 46) }\end{array}$ & p value \\
\hline Hypertension & & & \\
\hline Yes & 12 & 17 & 0.0019 \\
\hline No & 2 & 29 & \\
\hline Hyperlipidemia & & & \\
\hline Present & 6 & 12 & 0.31 \\
\hline Absent & 8 & 34 & \\
\hline CKD & & & 0.5 \\
\hline Present & 5 & 4 & \\
\hline Absent & 9 & 42 & \\
\hline Smoking & & & 0.14 \\
\hline Yes & 4 & 24 & \\
\hline No & 10 & 22 & \\
\hline HbA1c & & & 0.005 \\
\hline $7-9 \%$ & 6 & 33 & \\
\hline$>9 \%$ & 8 & 6 & \\
\hline Serum Albumin & & & \\
\hline$<3.5$ grams & 12 & 21 & \\
\hline$\geq 3.5$ grams & 2 & 25 & \\
\hline Etiology & & & \\
\hline Neuropathy & 3 & 17 & \\
\hline Ischemic & 2 & 13 & \\
\hline Neuro-ischemic & 9 & 42 & \\
\hline Bone changes & & & \\
\hline Present & 8 & & \\
\hline Absent & 6 & & \\
\hline & & & \\
\hline
\end{tabular}

\section{DISCUSSION}

Age range (36-70 years) and male: female (3:1) ratio was comparable with other studies. The incidence is more among males probably as they are mostly working outdoor, which makes them more vulnerable for trauma and sequel [4]. Incidence of gangrene (16 cases) in the present series is comparatively equal to that of Pennsylvania series [5]. In this study $33.3 \%$ of cases of diabetic foot ulcer have been found to be of neurogenic origin [5], $15 \%$ 
Arijit Singha Mahapatra \& Pritam Mitra., SAS J Surg, Mar, 2021; 7(3): 124-129

ischemic and 25\% neuro-ischemic. Other study revealed neuropathy was the etiological factor for more than $60 \%$ DFU [6]. In this study the sites of ulcers also corroborates with the etiology of the ulcers. The mostcommon site was foot margin, followed by plantar surface of metatarsal head and toes tip.

In the present study, raised serum glycosylated hemoglobin, elevated serum lipid profile, impaired renal function, h/o poor foot wear, limb deformity and limited joint mobility have significantly affected DFU $[7,8]$. It is worth mentioning that these clinicpathological parameters have been chosen by extensive search from standard literatures and text books.

A significant association however was found with regard to tobacco usage either in smoked or chewed form. There were a total of 28 tobacco users, of these 20 individuals (66.67\%) showed presence of PVD. This when compared to only 10 out of 32 nonsmokers showing presence of PVD was significant [9, 10]. This correlates with existing data where in tobacco as an independent factor is implicated in the etiology of PVD and also seen to accelerate changes in diabetic individuals

In our study, history of poor footwear use has been found in $33.3 \%$ cases of diabetic foot ulcers. In our institute (Govt. Hospital), as the majority of patients attending the OPD and getting admitted, belong from the low socio-economic status group have poor footwear use [11]. A US based study suggested that individuals with diabetic foot ulceration were 5.1 times more likely to have poorly fitting shoes than those without a wound [12].

Our study shows a prevalence of $10 \%$ cases of associated deformity of the limb among the admitted patients with diabetic foot ulcers. A study in NorthWest England has shown $1.57 \%$ cases of foot deformity in patients with DFU [13]. The percentage in our study is a bit higher which may be due to the higher percentage of pure and mixed neurogenic ulcers are present in our part in the setting of a poor socioeconomic and poor awareness.

In the present study, it was found the percentage of patients with DFU, having higher HbA1c level (>9) is to be $23.3 \%$.Several studies have shown that there may be strong association between impaired renal function and DFU [14]. In the present study, 15\% of all patients having DFU, have impaired renal function and 3 patients (5\%) had history of dialysis also. In one study, serum cholesterol has been mentioned to be a possible risk factor of DFU. It shows serum cholesterol to be raised in $4.75 \%$ cases of neuropathic, $5.23 \%$ cases of ischemic and $4.84 \%$ cases of neuro-ischemic foot ulcers. In our study, elevated lipid profile has been found in $30 \%$ cases of DFU. The biochemical abnormality has been found in 7 cases
$(38.89 \%)$ of neurogenic, 4cases $(22.23 \%)$ of ischemic and 7 cases $(38.89 \%)$ of neuro-ischemic ulcers.

In the present study the commonest organism cultured was staphylococcus aureus16(26.7\%) which was similar to study conducted by others showed 12 patients (20) hadpolymicrobial growth pattern [15].

The prevalence of PVD was found to be $50 \%$ with 30 out of 60patients showing vascular compromises diagnosed by arterial Doppler study. The prevalence in males was found to be $54.35 \%$ while in females was $35.7 \%$. The distal lower limb vessels were found to be more involved with $90 \%$ having lesions in the anterior tibial, posterior tibial or both. This correlates with existing literature which shows that distal small arteries are more involved in diabetics [16].

The severity of the diabetic foot infection and outcomes were also significantly worse in individuals with PVD [17] Of the 16 patients with gangrene either of the toe or forefoot requiring some sort of amputation or other intervention $12(75 \%)$ were found to have PVD. On the basis of this study there is a relevance of investigating the presence of peripheral vascular disease and the need to do it on a routine basis even in apparently asymptomatic individuals can be advocated.

In our study, we have followed up these 60 cases for 6 months and divided them into two groups according to the outcome of the ulcers after treatment. We have seen 46 cases $(76.7 \%)$ healed completely by that time, but 14 cases $(23.3 \%)$ were non-healing ulcers. So we have tried to figure out the clinical, biochemical, and radiological factors, that are affecting the outcomes in those cases. Among the 14 cases of non-healing ulcers, all the patients were more than 40 years of age group and the incidence of non-healing ulcers are increasing with age. There is a statistical significant association of non-healing ulcer with advanced age. It also can be explained as the extremes of age have low general body immunity. While studying the occurrence of the non-healing ulcers according to the sex, we have found out that out of the 14 patients, 10 patients were male and 4 were female. In our study, 16 patients were presented with initial gangrene and out of them, 10 had developed non healing ulcers on further follow up. So, there is definite statistical significance of initial clinical presentation like gangrene with the healing status of the ulcers. Sites of ulcer have no role to play in healing, as per our study. Among the 14 non healing ulcers, 12 patients were hypertensive. So, there is a statistical association of healing of DFUs with blood pressure. Hypertension is definitely a predictor of poor outcome of DFUs in our study. Chronic kidney disease is frequently associated with diabetes. But, diabetic nephropathy has no role to play in healing of DFUs, as there is no statistical significance found between them in my study. Smokers are not more prone to develop non healing ulcers, as in the present study, no 
Arijit Singha Mahapatra \& Pritam Mitra., SAS J Surg, Mar, 2021; 7(3): 124-129

association found between them. Ulcer healing in DFUs does not depend on the etiology. In this study, no statistical association found between etiopathogenesis and ulcer healing. Diabetic status is significantly associated with the healing of the ulcers. A significant association is found in this present study between HbA1c and healing ofthe ulcers. DFUs with uncontrolled diabetes are more likely to not heal. There are many studies supporting the role of serum albumin in ulcer healing. In the present study also, a significant association found between hypoalbuminemia and nonhealing ulcers. The patients of DFUs with bone changes are not likely to heal easily. So, bone involvement is definitely an indicator of poor outcome.

Not all diabetic foot complications can be prevented, but it is possible to dramatically reduce the incidence through appropriate management and prevention programs. The multidisciplinary team approach to diabetic foot disorders has been demonstrated as the optimal method to achieve favourable rates of limb salvage in the high-risk diabetic patient. Good glycemic control will prevent acute complications such as DKA and hypoglycemia.

\section{CONCLUSION}

In the present study, some factors have emerged as predictors of poor outcome of the DFUs like old age, association with gangrene, hypertension, uncontrolled diabetes, hypoalbuminemia, and bone involvement. So, with the help of these factors we can predict the outcome beforehand, and as there are some modifiable factors, we can individualize the treatment for them.

\section{REFERENCES}

1. Kerr M. Foot care for people with diabetes: The Human and Financial Cost. NDFA. 2016.

2. Margolis DJ, Malay DS, Hoffstad OJ. Incidence of diabetic foot ulcer and lower extremity amputation among Medicarebeneficiaries, 2006 to 2008. Data Points Publications Series 2011.

3. Nather A, Bee CS, Huak CY, Chew JL, Lin CB, Neo S, Sim EY. Epidemiology of diabetic foot problems and predictive factors for limb loss. J Diabetes Complications. 2008; 22: 77-82.

4. International Working Grouponthe Diabetic Foot. InternationalConsensuson the Diabetic Foot.
Amsterdam; 2004. 1-96.

5. Abbott CA. The North West Foot Care. Diabetic Med. 2002; 342:34-8.

6. Schaper NC. Diabetic foot ulcer classification system for research purpose: a progress report on criteria on including patients in research studies. Diabetes Metab Res Rev. 2004; 20:90 -5.

7. Akbari CM, Macsata R, Smitth BM, Sidawy AN. Overview of the diabetic foot. Semin Vase Surg. 2003; 16:3-11.

8. Akbar N, Bilal N. Relation of glycemic control with Diabetic Foot Lesions. Intr J Path. 2004; 2: 90-3.

9. Armstrong DG, Lavery LA, Harkless LB. Validation of diabetic wound classification system. The contribution of depth infection and ischemic risk of amputation. Diabetes Care. 1998; 21:855-9.

10. Lipsky BA, Berendt AR, Deery HG, Embil JM, Joseph WS, Kachmer AW. Diagnosis and treatment of diabetic foot infections. Clin Infect Dis. 2004; 39:885-910.

11. Viswanathan V, Snehalatha C. Association of limited joint mobility and high plantar pressure in diabetic foot ulceration in Asian Indians. Diabetes Res Clin Pract. 2003; 60: 57-61.

12. Nayamu PN, Otieno CF. Risk Factors and prevalence of Diabetic foot ulcers at Kenyatta National Hospital, Nairobi. East Afric Med J. 2003; 80:1223-9.

13. Khamash MR, Obeidt KA. Prevalence of ischemia in diabetic foot infection. World J Surg. 2003; 27:797-9.

14. Margolis DJ, Hofstad O. The association between Renal Failure and Foot Ulcer or lower extremity amputation in those patients with diabetes. Diabetes Care. 2008; 31:1331-6.

15. Zimmerli W, Fluckiger U. Classification and microbiology of osteomyelitis. Orthopade. 2004; 33:267-72.

16. Fryker RG, Zgonis T. The diabetic foot: Lower exteamity arterial disease limb salvage. Sidawy LN. Lippincot Williams and Wilkins; Philadelphia: 2006: 117-128.

17. Belkin M, Detal C. Peripheral arterial occlusive disease. Townsend CM, Beauchamp RD. Sabistons Textbook of Surgery. $18^{\text {th }}$ ed. Philadelphia; 2008; 1945-7. 